\title{
Producción de conocimiento geométrico mediante la geometría del doblado de papel ${ }^{1}$
}

\author{
Geometrical knowledge production by paper folding geometry
}

Produção de conhecimento geométrico por geometria dobradura de papel

Recibido: mayo de 2013

Aceptado: agosto de 2013
Zaida Margot Santa Ramírez ${ }^{2}$

Carlos Mario Jaramillo López ${ }^{3}$

\section{Resumen}

Este trabajo de investigación, mediante un estudio de casos cualitativo, pretende determinar cómo un colectivo de estudiantes produce conocimiento geométrico cuando interactúa con la geometría del doblado de papel. De esta manera, se espera mostrar que la herramienta se convierte en un medio que posibilita la producción de conocimiento geométrico, es decir, se busca caracterizar el sistema "Seres-humanos-con-doblado-depapel”, como una extensión del constructo teórico Humans-with-media fundamentado por Borba \& Villarreal (2005).

Palabras clave: Producción; matemáticas escolares; geometría; geometría euclídea; conocimiento geométrico; aula; recursos didácticos; materiales manipulativos; doblado de papel; Humans-with-media.

\section{Abstract}

This research, using a qualitative case study aims to determine how a student group produces geometric knowledge when it interacts with the geometry of the folded paper. Thus, it is expected to show that the tool becomes a medium that enables the production of geometric knowledge, that is, to characterize the system "-human-beings with de-folded paper", as an extension of the construct theoretical Humans-with-media founded by Borba \& Villarreal (2005).

Keywords: Production; school mathematics, geometry, Euclidean geometry, geometrical knowledge, classroom teaching resources, manipulatives, paper folding; Humans-with-media.

\section{Resumo}

Esta pesquisa, através de um estudo de caso qualitativo tem por objetivo determinar como um grupo de estudantes produz conhecimento geométrica quando ele interage com a geometria do papel dobrado. Assim, espera-se para mostrar que a ferramenta se torna um meio que permite a produção de conhecimento geométrico, isto é, para caracterizar o sistema de "seres humanos-com papel de dobrado", como uma extensão do

\footnotetext{
1 Este artículo surge del trabajo de investigación "Producción de conocimiento geométrico mediante la geometría del doblado de papel”, que se está desarrollando en el marco del programa Doctorado en Educación, de la Facultad de Educación de la Universidad de Antioquia.

2 Universidad de Antioquia. Medellin, Colombia. Contacto: zsanta@ayura.udea.edu.co

3 Universidad de Antioquia. Medellin, Colombia. Contacto: cama@matematicas.udea.edu.co
} 
construto Os seres humanos-com-mídia teóricas fundadas por Borba e Villarreal (2005).

Palavras-chave: Produção; matemática escolar, geometria, geometria euclidiana, o conhecimento geométrico, recursos didáticos em sala de aula, manipuláveis, dobradura de papel; Seres humanos-com-mídia.

\section{Presentación del problema}

Planteamiento del problema. $\mathrm{Mi}$ experiencia como maestra de geometría en todos los grados de la básica secundaria, me permite afirmar que los estudiantes tienen dificultades en la comprensión de conceptos geométricos; en particular he notado grandes problemas cuando intentan definir lugares geométricos como mediatriz, bisectriz, circunferencia, rectas paralelas, rectas perpendiculares, secciones cónicas, entre otros. Por otro lado, mi experiencia docente a nivel universitario, en especial en el Seminario Complementario I, del Programa de Maestría en Regiones de la Universidad de Antioquia (primer semestre de 2012), me ha mostrado que algunos maestros de las regiones desconocen los conceptos relacionados con los lugares geométricos. De hecho, en un taller de doblado de papel que realicé con ellos, me encontré con expresiones como: "No conocemos qué es un lugar geométrico"; "profe, nosotros desconocíamos que la mediatriz y la bisectriz se definieran como lugares geométricos", entre otras.

Al igual que los autores Cruz \& Mariño (1999) y Mata (2006), que encontraron que los estudiantes tienen dificultades en la comprensión de conceptos geométricos, específicamente aquellos relacionados con las secciones cónicas, en Santa (2011) encontré que: "muchos estudiantes, de la interfase bachillerato - universidad, tienen dificultades para comprender los conceptos de las secciones cónicas como lugares geométricos, mientras que se les facilita la búsqueda algorítmica de sus ecuaciones" ( p. 57). Para solucionar parte de este problema, en dicho estudio me centré en la descripción de la comprensión individual del concepto de elipse como lugar geométrico, en los estudiantes del grado décimo de una Institución Educativa pública de la ciudad de Medellín. Sin embargo, inferí que esa comprensión dependía notablemente de las interacciones del estudiante, con la geometría del doblado de papel y con la investigadora. En este sentido, parecía que la producción de conocimiento, en particular conocimiento geométrico, se daba mediante la interacción de un colectivo de seres humanos con un medio determinado, que era el doblado de papel; pero dicha inferencia no fue objeto de estudio en la investigación a nivel de maestría y, en ese entonces, pasó a un segundo plano.

Considerando las ideas anteriores, con mi estudio doctoral pretendo lograr que un colectivo de estudiantes produzca conocimiento geométrico (todos los conceptos que surjan de los Axiomas de Huzita - Hatori (Santa, 2011)) a través de actividades basadas en la geometría del doblado de papel. De esta manera, espero mostrar que esta herramienta se convierte en un medio que posibilita la producción de conocimiento geométrico, es decir, pretendo caracterizar el sistema "Seres-humanoscon-doblado-de-papel”, como una extensión del constructo teórico Humans-with-media fundamentado por Borba \& Villarreal (2005).

Pregunta de investigación. De acuerdo con el planteamiento del problema, con el estudio se busca responder la siguiente pregunta de investigación:

¿Cómo un colectivo de estudiantes produce conocimiento geométrico al interaccionar con la geometría del doblado de papel?

\section{Marco de referencia conceptual}

Humans-with-media. Este constructo teórico se fundamenta en dos ideas principales: la primera es que "la cognición no es un trabajo individual sino más bien de naturaleza colectiva" (Villa-Ochoa \& Ruiz, 2010, p. 518) y la segunda, que la construcción del conocimiento incluye "herramientas, dispositivos, artefactos y medios" (p. 518). Por lo tanto, Borba \& Villarreal (2005) afirman que no tiene mucho sentido hablar de una separación entre los seres humanos y los medios, dado que para ellos, los medios hacen parte esencial y fundamental del sujeto e, incluso, lo componen y constituyen. En 
este sentido, Levy (1993) enfatiza que la historia de los medios siempre estará entrelazada con la historia de la propia humanidad (Citado por Borba, 2012, p. 138).

Por otro lado, estos autores afirman que el uso de diferentes tipos de medios puede conducir a la producción de diferentes tipos de conocimientos en colectivos diferentes. Es decir, sostienen que "una nueva tecnología resulta en un nuevo colectivo que produce nuevos conocimientos, que son cualitativamente diferentes a los conocimientos producidos por otros colectivos" (Borba \& Villarreal, 2005, p. 24).

En este sentido, Borba (2002) afirma que "la construcción del conocimiento se logra a partir de un colectivo compuesto por humanos y no humanos" (p. 142). En particular, este autor enfatiza en "el papel que los medios tienen, como actores no humanos, en moldear las posibilidades que tienen esos colectivos de construir conocimiento" (p. 142). Por esta razón, este constructo teórico adopta una perspectiva de carácter teórica que "apoya la idea de que el conocimiento es producido por un colectivo compuesto de seres humanos-con-medios, o seres-humanos-con-tecnologías, y no como otras teorías sugieren, por los seres humanos individuales o, colectivos compuestos solo por seres humanos" (Borba \& Villarreal, 2005, p. 23).

De acuerdo con esta postura teórica, el conocimiento siempre ha estado condicionado por distintos medios en toda la historia de la humanidad. Como consecuencia de ello, las personas producen conocimientos de diferentes maneras, en concordancia con el uso de diferentes tecnologías. Por lo tanto, siguiendo a Levy (1993), es posible intervenir de manera consciente en la forma en que esta tecnología permea la vida de los seres humanos (Citado por Borba \& Villarreal, 2005). De la misma manera se podría pensar en la Geometría del doblado de papel, como un medio que puede dar forma determinada al conocimiento geométrico en un grupo de estudiantes, si el maestro interviene de manera consciente en esta relación dialógica.

Visualización. Borba \& Villarreal (2005) consideran que la visualización es una forma de razonamiento en la investigación en matemáticas y, en particular, en Educación Matemática. Ellos presentan dos niveles en los que la visualización se puede considerar: en primer lugar, "asociado a su uso en la prueba matemática formal" (p. 86) y, en segundo lugar, "relacionado con su uso en otras actividades matemáticas tales como la elaboración de conjeturas, la solución de problemas o los intentos de explicar algunos resultados matemáticos a colegas o estudiantes" (p. 86). Para estos autores, la visualización se considera importante, por las siguientes razones:

“...constituye una forma alternativa de acceder al conocimiento matemático; la comprensión de los conceptos matemáticos requiere múltiples representaciones y la representación visual puede transformar la comprensión de sí mismo; la visualización es parte de la actividad matemática y una manera de resolver problemas..." (Borba \& Villarreal, 2005, p. 96).

\section{Metodología}

El estudio propuesto está orientado bajo un paradigma de corte cualitativo, por las razones que se enuncian a continuación:

- Las actividades se diseñan de acuerdo con el contexto del colectivo de estudiantes. En este sentido, se considera fundamental tener presente la historicidad del grupo de estudiantes y su aspecto subjetivo de la realidad, para poder llegar a caracterizar el sistema "Seres-humanos-con-doblado-de-papel" en la particularidad inicial de los participantes.

- Las interacciones entre el colectivo de estudiantes con la geometría del doblado de papel y con el investigador, son las que permiten caracterizar el sistema "Seres-humanos-condoblado-de-papel”, que dependerá del contexto del que se extraiga la información.

- Se pretende comprender la realidad tanto en su lógica interna como en su especificidad (Sandoval, 2002).

- La investigación se fundamenta en un proceso inductivo, en la que se pretende "explorar y describir, y luego generar perspectivas teóricas" (Hernández, Fernández \& Baptista, 2006, p. 8).

- Análisis de la información 
- La información se recolectará a través de las siguientes técnicas:

- Observaciones: Se realizarán varias observaciones al colectivo de estudiantes, cuando estén desarrollando actividades mediante la geometría del doblado de papel, en el estudio de los Axiomas de Huzita - Hatori (Santa, 2011). Lo que se pretende analizar son las interacciones del grupo al utilizar este medio en la producción de conocimiento geométrico.

- Revisión del material de los estudiantes que surja de todas las actividades propuestas.

- Entrevistas individuales y grupales (discusiones en grupo o interacción e introspección con grupos), para analizar las interacciones dentro del grupo de estudiantes con el medio de la geometría del doblado de papel.

- Dicha información será analizada mediante un proceso de triangulación entre las observaciones del investigador, el constructo teórico y la información recolectada (a través de observaciones, revisiones documentales y entrevistas), con miras a caracterizar el sistema Seres-humanos-con-doblado-de-papel, como una extensión del constructo teórico Humans-with-media.

\section{Conclusiones}

Se espera que el paso de lo concreto a lo abstracto, de representaciones externas a internas, de lo manipulable a lo simbólico, se logre a través de la interacción de un colectivo de estudiantes con el medio de la geometría del doblado de papel (actividades basadas en construcciones hechas mediante el doblado). Las entrevistas diseñadas tendrán un tinte de carácter socrático y se espera que, mediante la visualización, se alcance la producción de conocimiento geométrico.

Además, con el estudio se pretenden alcanzar los siguientes resultados:

- Caracterizar el sistema "Seres-humanoscon-doblado-de-papel" como extensión del constructo teórico de Humans-with-media.
- Producción de conocimiento geométrico de un colectivo de "Seres-humanos-con-doblado-de-papel".

- Hacer aportes a las ideas de visualización, expuestas en el constructo teórico en cuestión, involucrando no sólo el sentido visual, sino el táctil en la construcción de representaciones mentales.

\section{Referencias}

Borba, M. (2002). Colectivos seres-humanos-com-mídias e a producao de Matemática. En: I Simposio Brasileiro de Psicología de Educación matemática.

Borba, M. \& Villarreal, M. (2005). Humans-withMedia and the reorganization of Mathematical Thinking. New York: Springer.

Cruz, L. \& Mariño, M. (1999). Sistema computarizado para la enseñanza de las secciones cónicas. Revista Educación, (97), pp. 14 - 21.

Hernández, R., Fernández, C. \& Baptista, P. (2006). Metodología de la Investigación. México: McGraw Hill.

Mata, F. (2006). Análisis sobre el razonamiento en el aprendizaje De los conceptos de la geometría analítica: el Caso particular de las secciones cónicas aplicando el modelo de Van Hiele. Tesis para obtener el grado de Maestro en Ciencias en Matemática Educativa. México: Instituto Politécnico Nacional.

Sandoval, C. (2002). Investigación cualitativa. Colombia: ARFO. Recuperado el 20 de abril de 2010, de http://contrasentido.yukei.net/ wp-content/uploads/2007/08/modulo4.pdf

Santa, Z. (2011). La elipse como lugar geométrico a través de la geometría del doblado de papel en el contexto de Van Hiele. Trabajo de investigación para optar al título de Magíster en Educación. Medellín: Universidad de Antioquia.

Villa-Ochoa J. \& Ruiz M. (2010). Pensamiento variacional: seres-humanos-con-GeoGebra en la visualización de nociones variacionales. En: Revista Educ. Matem. Pesq., São Paulo, 12, (3), pp. $514-528$. 